\title{
A multicenter phase III study comparing Simultaneous Integrated Boost (SIB) radiotherapy concurrent and consolidated with S-1 versus SIB alone in elderly patients with esophageal and esophagogastric cancer - the 3JECROG P-01 study protocol
}

Chen $\mathrm{Li}^{1+}$, Xiaomin Wang ${ }^{2+}$, Xin Wang ${ }^{1+}$, Chun $\mathrm{Han}^{3}$, Ping Wang ${ }^{4}$, Qingsong Pang ${ }^{4}$, Junqiang Chen ${ }^{5}$, Xinchen Sun ${ }^{6}$, Lan Wang ${ }^{3}$, Wencheng Zhang ${ }^{4}$, Yu Lin ${ }^{5}$, Xiaolin Ge ${ }^{6}$, Zongmei Zhou' ${ }^{1}$ Wenjie Ni ${ }^{1}$, Xiao Chang ${ }^{1}$, Jun Liang ${ }^{7}$, Lei Deng ${ }^{1}$, Wenqing Wang ${ }^{1}$, Yidian Zhao ${ }^{2^{*}}$ and Zefen Xiao ${ }^{1 *}$ (D)

\begin{abstract}
Background: The importance of definitive radiotherapy for elderly patients with esophageal and esophagogastricjunction cancer is pronounced. However, little is known in terms of the best way to combine radiotherapy with other treatment options. This study aims to compare the efficiency of SIB radiotherapy alone with SIB radiotherapy concurrent and consolidated with S-1 for elderly patients. Comprehensive geriatric assessment is also incorporated in the procedure of treatment.

Methods/design: The study is a two arm, open, randomized multicenter Phase III trial with patients over 70 years old with stage IIA-IVB (UICC 2002, IVB only with metastasis to supraclavicular or celiac lymph nodes) squamous cell carcinoma or adenocarcinoma of esophagus or gastroesophageal junction. A total of 300 patients will be randomized using a 1:1 allocation ratio stratified by disease stage and study site. Patients allocated to the SIB arm will receive definitive SIB radiotherapy (95\%PTV/PGTV 50.4Gy/59.92Gy/28f) while those randomized to SIB + S-1 arm will receive definitive SIB radiotherapy concurrent and consolidated with S-1. The primary endpoint of the trial is 1-year overall survival. Secondary objectives include progression-free survival, recurrence-free survival (local-regional and distant), disease failure pattern, toxicity profile as well as quality of life. Besides, detailed radiotherapy protocol and quality assurance procedure have been incorporated into this trial.
\end{abstract}

Discussion: The proportion of elderly patients in esophageal cancer is now growing, but there is a lack of evidence in term of treatment standard for this group of patients, which is what we aim to obtain through this prospective phase III study.

\footnotetext{
*Correspondence: ayydz101@163.com; xiaozefen@sina.com

${ }^{\dagger}$ Chen Li, Xiaomin Wang and Xin Wang contributed equally to this work. ${ }^{2}$ Department 4th of Radiation Oncology, Anyang Cancer Hospital, Anyang 455000, China

${ }^{1}$ Department of Radiation Oncology, National Cancer Center/National Clinical Research Center for Cancer/Cancer Hospital, Chinese Academy of Medical Sciences and Peking Union Medical College, Beijing 100021, China Full list of author information is available at the end of the article
}

(c) The Author(s). 2019 Open Access This article is distributed under the terms of the Creative Commons Attribution 4.0 International License (http://creativecommons.org/licenses/by/4.0/), which permits unrestricted use, distribution, and reproduction in any medium, provided you give appropriate credit to the original author(s) and the source, provide a link to the Creative Commons license, and indicate if changes were made. The Creative Commons Public Domain Dedication waiver (http://creativecommons.org/publicdomain/zero/1.0/) applies to the data made available in this article, unless otherwise stated. 
(Continued from previous page)

Trial registration: clinicaltrials.gov NCT02979691. Registered November 22, 2016.

Keywords: Esophageal cancer, Esophagogastric junction cancer, Elderly, Concurrent chemoradiotherapy, Definitive chemoradiotherapy, Consolidated chemotherapy, Simultaneous integrated boost, Intensity modulated radiotherapy, Randomized controlled trial, S-1, Comprehensive geriatric assessment

\section{Background}

Esophageal and esophagogastric-junction cancer (EC and EGJC) is the fourth most common cancer in China and elderly EC/EGJC patients accounts for approximately $30 \sim 40 \%$ of all cases. $[1,2]$. The management of elderly patients with EC/EGJC is still challenging as their relative poor physical conditions impose great limitations on their treatment. Generally, they are considered ineligible for esophagectomy, the major component of treatment norm for patients with resectable EC/EGJC, because of the high rates of postoperative morbidity and mortality (especially those received preoperative chemoradiation) [3, 4]. A promising alternative without major toxicity is definitive chemoradiotherapy (dCRT), which is initially designed for patients with locally advanced EC/EGJC. However, the dual-drug intravenous chemotherapy regimen (fluorouracil/capecitabine + platinum drugs) recommended by National Comprehensive Cancer Network (NCCN) [5] and European Society for Medical Oncology (ESMO) [6] may bring severe acute and late toxic effects and poor compliance rate in elderly population. Thus, a modified dCRT regimen specific to the elderly is in great demand.

In this paper, we propose a prospective phase III clinical trial to improve dCRT regimen for elderly patients in three aspects: First, S-1, a single chemotherapeutic drug will be used instead of the conventional two-drug chemotherapy regimen to improve therapeutic effect while maintaining the rate of toxicities at a relatively low level. Secondly, SIB technique will be adopted to increase the dose of regions at high risk, while simultaneously reduce the dose of organs at risk (OAR). The adopted radiation dose pattern come from a prospective phase I/II trial previously conducted in our center [7]. Finally, the specialty of elderly population will be considered by integrating comprehensive geriatric assessment (CGA) into treatment procedure.

CGA, a multidisciplinary evaluation of the elderly, usually covers functional status, cognitive capacities, emotional status, comorbidities, nutritional status, social and environmental situations, and a possible geriatric syndrome. Over the past decade, the CGA has been proposed as a tool for managing elderly patients with cancer [8-14]. However, no study has ever attempted to incorporate CGA to the treatment of patients with EC/EGJC. 3JECROG P-01 trial is the first large-scale prospective phase III study to combine CGA with the treatment of elderly EC/EGJC patients to the best of our knowledge.

To summarize, this prospective, multi-center phase III clinical trial is initiated in the expectation of obtaining high-level type I evidence for the standard treatment of EC/EGJC among elderly patients.

\section{Methods/design}

Study design and objectives

This study is an open, multicenter Phase III clinical trial. Approximately 15 participating centers throughout China are involved. The technique of SIB is adopted in this study with a dose of $50.4 \mathrm{~Gy} / 2.14 \mathrm{~Gy} / 28 \mathrm{f}$ to planning target volume (PTV) and 59.92Gy/2.14Gy/28f to planning gross tumor volume (PGTV). S-1 is given both concurrent with and after radiotherapy. Patients enrolled are stratified by disease stage and study site and assigned to either SIB + S-1 group or SIB group using a 1:1 allocation ratio at randomization. A flow chart giving an overview of the study design is shown in Fig. 1.

The primary objective of this trial is to compare the 1-year OS of SIB + S-1 group with SIB group. Secondary objectives include progression-free survival, recurrence-free survival (local-regional and distant), disease failure pattern, toxicity profile as well as quality of life.

The study started on February, 2017 and the duration of inclusion will be approximately 2 and a half years.

\section{Patient \\ Patient selection}

Patients older than 70 years old with histocytologically proven stage IIA-IVB (UICC 2002, IVB only with metastasis to supraclavicular or celiac lymph nodes) inoperable squamous cell carcinoma (SCC) or adenocarcinoma (AC) of esophagus or gastroesophageal junction are eligible for recruitment. No other treatment is allowed before enrollment. For cancer of esophagogastric junction, the center of the tumor could not extend $2 \mathrm{~cm}$ into the gastric cardia. Patients must have adequate hematological, renal and hepatic functions defined as: neutrophils $\geq 3.5 \times$ $10^{9} / \mathrm{L}$, granulocytes $\geq 1.5 \times 10^{9} / \mathrm{L}$, platelets $\geq 100 \times 10^{9} / \mathrm{L}$, urea nitrogen $(\mathrm{BUN}) \leq 1.0 \times$ upper normal limit (UNL), creatinine $(\mathrm{Cr}) \leq 1.0 \times$ upper normal limit (UNL), alanine aminotransferase (ALT), aspartate aminotransferase (AST) $\leq 1.5 \times$ UNL; alkaline phosphatase $(\mathrm{ALP}) \leq 1.5 \times$ UNL; total bilirubin $\leq \mathrm{UNL}$. General condition of enrolled patients 


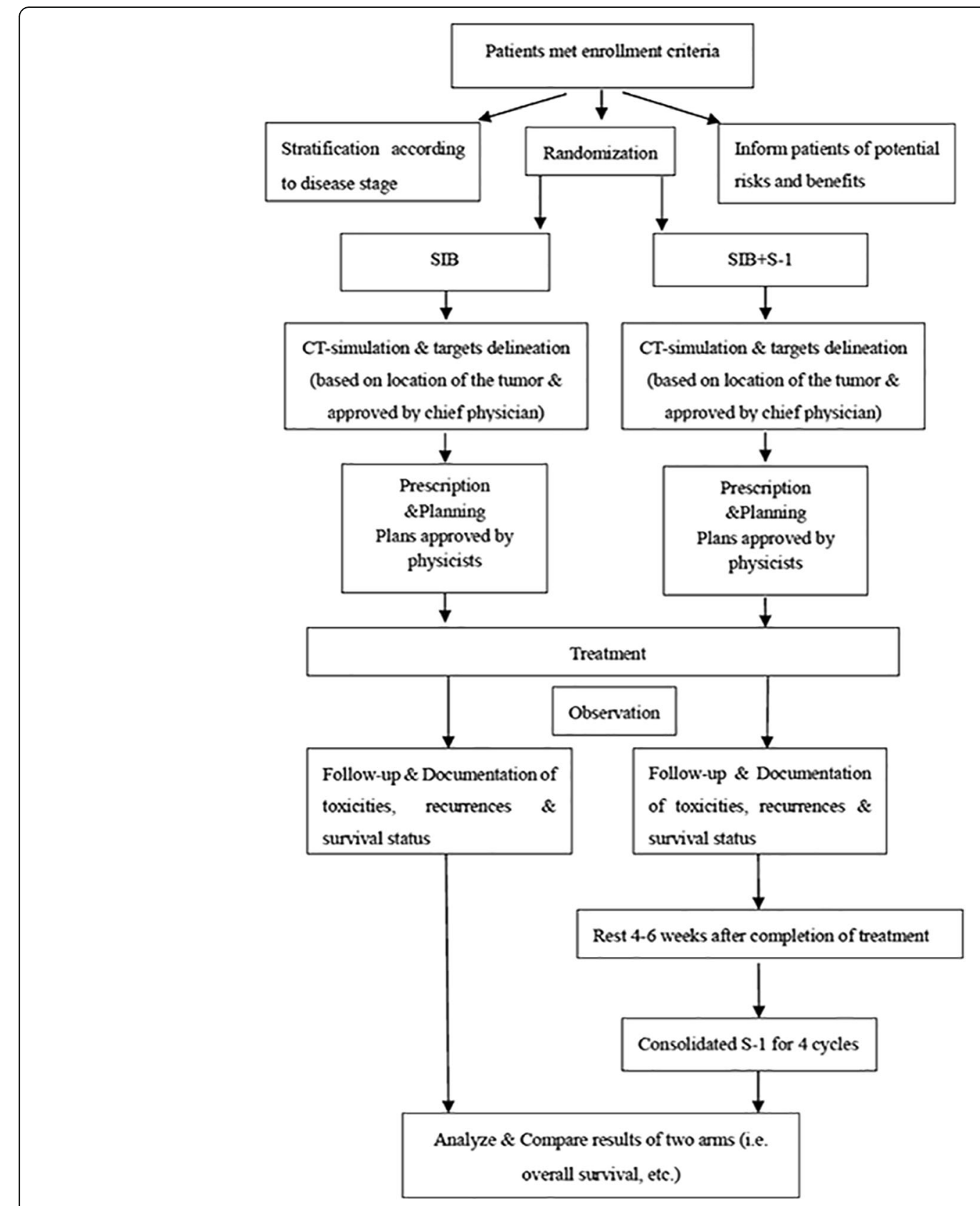

Fig. 1 Flow chart of 3JECROG P-01

must be acceptable (i.e. Karnofsky Performance Status $\geq 70$ or Eastern Cooperative Oncology Group performance status $\leq 1$, Charlson Comorbidity Index $\leq 3$ ).

Exclusion criteria include prior malignancies (other than curable non-melanoma skin cancer or in situ cervical cancer) within 5 years, distant lymph node (other than metastasis to supraclavicular or celiac lymph nodes) or visceral metastasis (including metastasis to bone, lung, liver, pleura or peritoneum, etc.), uncontrolled infection such as tuberculosis or hepatitis, uncontrolled hypertension or diabetes, severe comorbidities such as myocardial infarction, cerebral embolism or serious arrhythmia within 6 months, obvious sign of esophageal perforation or deep ulcer affirmed by barium esophagram.

\section{CGA}

As mentioned before, due to the specialty of elderly population, enrolled patients will receive a comprehensive assessment called CGA (including social support, general status, body function, nutritional status, mental health, cognitive ability and so forth) both before and 
after treatment. Detailed procedures of CGA are listed in Table 1.

\section{Radiotherapy}

For patients with lower-thoracic EC or EGJC, a series of pre-treatment procedure will be adopted to minimize the effect of stomach-volume-variation during treatment delivery. Patients should be fasted for at least $4 \mathrm{~h}$ and then drink $200-300 \mathrm{ml}$ semiliquid $15 \mathrm{~min}$ before CT simulation and daily irradiation. Supine position is performed with both arms straight beside the body. Head and neck hood is recommended for patients with cervical or upper-thoracic EC, while body film is used for middle, lower-thoracic EC and EGJC.

The Gross Tumor Volume (GTV-T) is defined as the primary tumor. The GTV-T will be determined using all available resources (physical examination, upper gastrointestinal contrast, endoscopy, EUS, CT-thorax/abdomen, MRI-thorax/abdomen, PET-CT, etc.).

The metastatic regional nodes (GTV-N) is defined as any lymph node diagnosed as or highly-suspected as metastatic.

As for the contouring of Clinical Target Volume (CTV), Involved-field radiotherapy (IFRT) is adopted.

Table 1 Domains and measures accessed by CGA before and after treatment

\begin{tabular}{|c|c|c|}
\hline Domain with measure & No. of items & Range of score \\
\hline \multicolumn{3}{|l|}{ Quality of life and specific module for EC } \\
\hline EORTC QLQ-C30 (Version 3.0) [34] & 30 & $30-126$ \\
\hline QLQ-OES-18 [35] & 18 & $18-72$ \\
\hline \multicolumn{3}{|l|}{ Functional status } \\
\hline ECOG performance status [36] & & $0-5$ \\
\hline Karnofsky performance status [37] & & $0-100$ \\
\hline Activities of daily life (Barthel index) [38] & 10 & $0-100$ \\
\hline $\begin{array}{l}\text { Instrumental activities of daily life } \\
\text { (Lawton's) [39] }\end{array}$ & 8 & $0-5 / 8$ \\
\hline \multicolumn{3}{|l|}{ Comorbidity } \\
\hline Charlson comorbidity index [40] & 19 & $0-37$ \\
\hline \multicolumn{3}{|l|}{ Cognitive status } \\
\hline Mini Mental State Examination [41] & 11 & $0-30$ \\
\hline \multicolumn{3}{|l|}{ Psychological status } \\
\hline Geriatric depression scale-5 items [42] & 5 & $0-5$ \\
\hline \multicolumn{3}{|l|}{ Nutritional status } \\
\hline \multicolumn{3}{|l|}{ Body mass index [43] } \\
\hline Mini-nutritional assessment [44] & 18 & $0-100$ \\
\hline \multicolumn{3}{|l|}{ Social support } \\
\hline $\begin{array}{l}\text { Medical Outcomes Study - social } \\
\text { support survey [45] }\end{array}$ & 20 & $20-100$ \\
\hline
\end{tabular}

CGA comprehensive geriatric assessment, EC esophageal cancer, ECOG Eastern Cooperative Oncology Group, EORTC The European Organization for Research and Treatment of Cancer, MOS Medical outcomes study
CTV consists of GTV-T plus a 0.6 to $0.8 \mathrm{~cm}$ circumferential margin, a $3 \mathrm{~cm}$ craniocaudal margin and GTV-N plus a $0.5 \mathrm{~cm}$ margin in all directions. For patients whose highest/lowest metastatic lymph nodes not exceeding $3 \mathrm{~cm}$ from the primary tumor, the upper/lower border of the is $3 \mathrm{~cm}$ superior/inferior to the primary tumor. For patients whose highest/lowest metastatic lymph nodes exceeding $3 \mathrm{~cm}$ from primary tumor, the upper/lower boundary of CTV is $0.5 \mathrm{~cm}$ superior/inferior to the furthest metastatic lymph node. No prophylactic irradiation is given to lymph node drainage regions. The PGTV is created by expanding GTV-T by $1.0 \mathrm{~cm}$ craniocaudally and $0.5 \mathrm{~cm}$ radially and GTV-N by a uniform $0.5 \mathrm{~cm}$ margin, and the PTV is derived from CTV plus a uniform $0.5 \mathrm{~cm}$ margin. The typical contouring of targets for tumor in different locations are depicted in Fig. 2 respectively.

The radiotherapy technique of SIB is performed 5 times a week. For thoracic esophagus, prescription dose is 95\% PTV/PGTV 50.40Gy/59.92Gy/28f. For EGJC, prescription dose is 95\% PTV/ PGTV 45.0Gy/53.5Gy/25f.

Both lungs, heart, spinal cord and spinal cord PRV should be contoured on the simulation images. For tumor of lower-thoracic EC and EGJC, physicians should also delineate stomach, liver, both kidneys and bowels (including small intestine and colon) as OARs. Bowels and spinal cord should be contoured $2 \mathrm{~cm}$ superior and inferior to PTV. OARs such as lungs, heart, stomach, kidneys and liver should be delineated from their upper border to their lower end. The volume of lung tissue receiving 20Gy or more should not exceed $28 \%$ of the total lung volume (V20 < 28\%). The mean dose of lung tissue should be lower than 16Gy (Dmean lung < 16Gy). Other dose constraints to OARs include: V40 heart < 30\%, V30 heart < 40\%, V40 stomach < 40\%, Dmax stomach < 55-60Gy, V40 small intestine $<40 \%$, Dmax small intestine <55Gy, V30 liver <30\%, V20 kidney <30\% and Dmax spinal cord PRV $<45$ Gy.

\section{Chemotherapy}

S-1 will be orally taken twice daily (or through nasal feeding tube for patients with enteral nutrition) within half an hour after meals during treatment days. It should not be taken during weekends or whenever radiotherapy is interrupted or stopped. The specific dosage for each patient is calculated according to body surface area in Table 2:

Generally, 4-8 weeks are needed for patients to recover after completion of SIB + S-1 (status of food intake, physical capacity, biochemical tests, etc.). Four cycles of S-1 will be given to those eligible for consolidation chemotherapy. Treatment schedule for patients in both arms is shown in Fig. 3. The daily-dose of consolidated S-1 is the same as that of concurrent phase, but it 


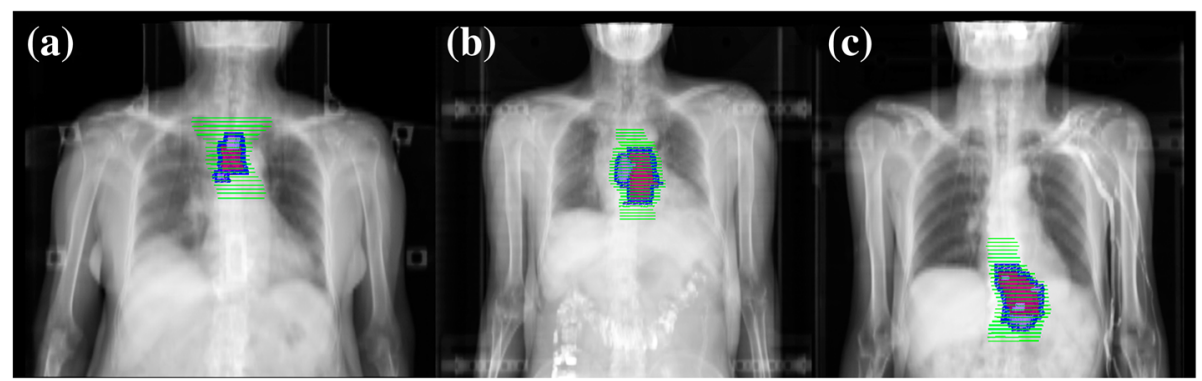

Fig. 2 Targets contouring of (a) upper thoracic esophagus (Ut); (b) middle thoracic esophagus (Mt); (c) lower thoracic esophagus (Lt). Red area indicates GTV-T, grey area includes GTV-N, blue area outlines PGTV and green area outlines PTV

will be administered for the first 2 weeks on each 21-days cycle. Blood routine should be monitored every week and hepatic and renal functions every cycle during chemotherapy.

\section{Toxicity and adverse events}

All treatment-related toxicities and adverse events should be graded according to the toxicity criteria of the Radiation Therapy Oncology Group (RTOG) and Common Terminology Criteria of Adverse Events (CTCAE) version 4.0 and recorded on patients' case report form (CRF) in detail. Serious Adverse Events (SAE) should be reported to the institutional ethical review committee in $24 \mathrm{~h}$ and dealt with properly.

S-1 should be terminated in case of Grade 2 anemia, thrombocytopenia, hepatic or renal dysfunction, Grade 4 leukopenia/neutropenia, Grade 3 radiation esophagitis and other non-homological Grade 3 toxicities. If adverse events de-grade to Grade $0-1$ within 1 week of drug withdrawal, patient can re-take S-1 at original dose, otherwise chemotherapy should be stopped henceforward. If Grade 3/4 radiation pneumonitis developed, both radiotherapy and oral intake of $S-1$ should be terminated. Consolidation of $\mathrm{S}-1$ will be re-assessed within 4-8 weeks after radiotherapy whichever grade of toxicities developed during dCRT.

\section{Follow-up}

Tumor regression should be evaluated according to RECIST Version 1.1 within 1-2 month after completion of treatment and rates of $\mathrm{CCR}$ and $\mathrm{CPR}$ should be documented. All patients should be followed up for at least 5 years after completion of the protocol and the time

Table 2 Relationship between body surface area and dose of S-1

\begin{tabular}{ll}
\hline Body surface area $\left(\mathrm{m}^{2}\right)$ & Initial dose \\
\hline$<1.25$ & $40 \mathrm{mg}$, bid \\
$\geq 1.25 \sim<1.50$ & $50 \mathrm{mg}$, bid \\
$\geq 1.5$ & $60 \mathrm{mg}$, bid \\
\hline
\end{tabular}

interval is every 3 months for the first 2 years, every 6 months for 3-5 years and once a year after 5 years.

Every follow-up should include: a. History-taking: symptoms of cough, fever, hoarseness, dysphagia or chest tightness, etc. b. Blood routine, basic metabolic panel, tumor markers, etc. c. Contrast enhanced CT of neck, thorax and abdomen, ultrasound of neck and abdomen, upper gastrointestinal contrast, bone scan (in case of bone pain or abnormally-elevated alkaline phosphatase), CT or MRI of brain (in case of any symptoms related to central nervous system), etc. d. Documentation of patients' status of survival, disease progression, subsequent treatment, nutrition, life quality and late toxic effects, etc.

\section{Statistical analysis \& sample size considerations}

We assume that an estimated difference in 1-y OS of $65 \%$ (SIB arm) versus $75 \%$ (SIB + S-1 arm) would justify applying this regimen in the future. Assuming a onesided significance level of 0.05 , a power of 0.80 , and $10 \%$ of lost in each arm, a total of 300 patients would be needed in this trial.

Estimates of median OS will be based on the Kaplan-Meier method and log-rank tests will be used to determine the significance. Cox regression analysis will be conducted to identify prognostic factors for survival benefit, which will be used in adjusted analyses of the treatment effect.

\section{Ethics}

The charge doctor should inform enrolled patients of the background of both treatment options, especially known efficiency and toxicities. It must be emphasized that the patient is allowed to refuse the treatment either before or during the study. Before enrollment, the patient's written consent should be obtained. The principal investigator (PI) will ensure that this study will be carried out in agreement with the "Declaration of Helsinki, Tokyo, Venice" or the laws and regulations of the country, whichever provides greater protection of the individual. The study has been approved by the institutional ethical review committee. 


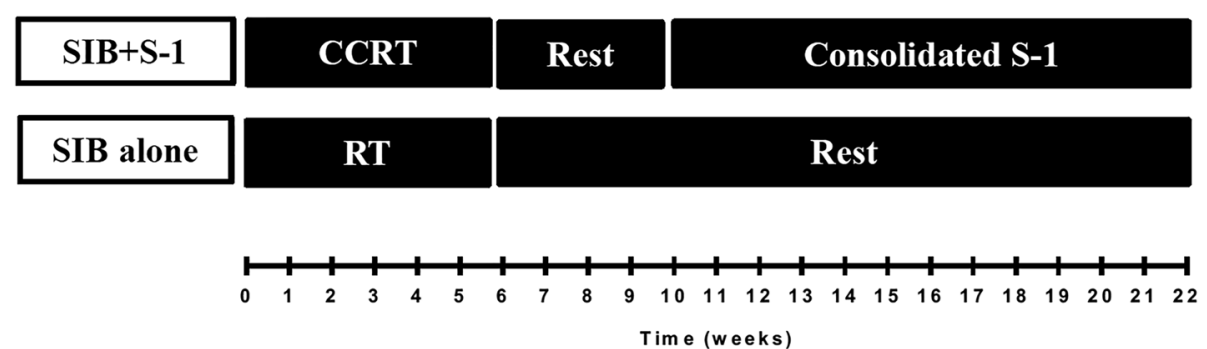

Fig. 3 Treatment schedule for both arms CCRT: Concurrent Chemoradiotherapy; RT: Radiotherapy

\section{Quality assurance}

A strict coordination and monitoring system has been constructed for this trial. First of all, a Radiotherapy Trials Quality Assurance (RTTQA) team consisted of physicians, dosimetrists, medical physicist and research fellows was built before the start-up of enrollment. Moreover, every participated branch-center should nominate at least one physician responsible for patient recruitment, data collection and online fill-out of CRF of their center. The RTTQA team will also name at least one censor to evaluate the quality of data collected from all branch centers and the censor is also in charge of keeping sound communication with physicians in charge from all participated centers.

Great effort has been put on assurance of treatmentquality and equality of all involved centers. An EC case was selected as an example by RTTQA team and distributed to all branch centers at the beginning of the study together with a brief clinical summary and CT imaging data of the selected case. All participated centers should send the case back to RTTQA team after completion of targets-delineation and RTTQA team would assess all collected cases for major and minor deviations. This is what we call the first round of Collection of Targets-Delineation (CTD). After that, the RTTQA team distributed a detailed protocol for targets-delineation to all branch-centers and let physicians in charge to contour targets again on the same sample case [15]. The countered case was collected again and this is what we call the second round of CTD. The second round of radiotherapy plans were also viewed thoroughly by the RTTQA team. It was proved that both quality and equality of the radiotherapy treatment improved significantly after distribution of the treatment protocol. This procedure assures that all centers and investigators have had a 'Pass' for the planned test case before entering patients into the trial. Censors from the RTTQA team will also randomly inspect the quality of treatment from branch centers during the study, on what occasion physicians in charge are required to provide a dataset of images, structures, radiotherapy plans and doses to the censor.

\section{Discussion}

In the proposed regimen, we replace the conventional two-drug chemotherapy regimen (fluorouracil/capecitabine + platinum drugs) by S-1, a single chemotherapeutic drug taken orally. The major consideration is to avoid the high toxic effects of the dual-drug chemotherapeutic regimen. In the study of RTOG 85-01 and INT-0123 [16, 17], although the enrolled population were mostly non-elderly patients under the age of 70 , dual-drug based chemoradiotherapy still brought serious adverse reactions. The rates of Grade 3-4 hematological toxicities were $48 \%$ vs $3 \%$ and that of gastrointestinal reaction were $33 \%$ vs $18 \%$, respectively. For elderly patients, worse results can be expected. Thus, the chemotherapeutic regimen from RTOG 8501 is generally modified for elderly patients in several small-sample prospective and retrospective studies. Generally, the toxicities remain at high levels even after these modifications if dual-drug chemotherapeutic regimen is adopted $[18,19]$. On the other hand, single-drug low-toxic chemotherapeutic regimen seems to be a promising treatment mode for elderly patients as they can reduce the toxic effects significantly. A prospective study evaluated the effect of radiation concurrent with single-drug agent (cisplatin/oxaliplatin) among patients older than 75 years. With a $53 \%$ cCR and $22 \% 3$-y OS, only $3 \%$ patients had grade $3 / 4$ hematological reactions during treatment [20]. In our study, S-1 is adopted as single concurrent and consolidated drug for its high efficiency and low toxicity. As a new generation of 5FU derivatives, S-1 showed better therapeutic effect than 5FU in some malignancies such as small cell lung cancer and pancreatic cancer [21]. It also led to significant lower rates of Grade 3/4 neutropenia ( $\mathrm{HR}=0.33,95 \% \mathrm{CI} \quad 0.25-0.44$ ) among patients with metastatic gastric cancer compared to 5FU [22]. Besides, S-1, a capsule preparation, provides a more convenient way of administration for elderly patients compared with continuous infusion of $5 \mathrm{FU}$.

Another highlight of our study is the use of SIB. Although the dose recommended by most guidelines $[5,6]$ is $50.4 \mathrm{~Gy}$, the local control rate (LCR) under $50.4 \mathrm{~Gy}$ is unsatisfying [23] and higher doses may be needed for 
high-risk areas. Besides, some retrospective study [24] have shown that in the subgroup of SCC, patients received high-dose irradiation ( $\geq 60 \mathrm{~Gy}$ ) had better OS and LCR than those who only received conventional dose (50.4Gy). Moreover, dosimetry studies have shown that for EC/EGJC, with the help of SIB, one can successfully increase the dose of boost areas without increasing the irradiation of OARs $[25,26]$. Thus this technique has been used more and more widely and most institutions have given boost areas a dose $\geq 60 \mathrm{~Gy}$. [27-30]. The dose pattern adopted in this trial (95\%PGTV/PTV 59.92Gy/ $50.40 \mathrm{~Gy} / 28 \mathrm{f}$, $\mathrm{EQD} 2=60.62 \mathrm{~Gy}$ ) is derived from a prospective Phase I/II trial previously conducted in our center [7]. According to that study, the use of SIB in dCRT patients is safe and feasible at 59.92Gy/50.40Gy/28f and the survival results are satisfying (1-y OS and local failure-free survival were 76.9 and $78.8 \%$ respectively). Therefore, we apply that dose model in this phase III study. To the best of our knowledge, this is the first large-scale prospective randomized study using SIB in the treatment of EC/EGJC.

Finally, 3JECROG P-01 is also the first multicenter prospective trial to incorporate CGA, a multidimensional diagnostic process investigating medical, psychosocial and physiological functions of the elderly, to the treatment of patients with EC/EGJC. It has been confirmed that the application of CGA in elderly patients with cancers can lower the rate of treatment-related complications, improve their QoL and body function and reduce the risk of hospitalization, etc. [31] Thus, CGA has been increasingly involved in the treatment of aged cancer patients worldwide [32, 33]. In this study, the relationship between CGA and survival status, the incidence of side effects and QoL in elderly patients will be analyze to explore the value of CGA in guiding the individualized treatment for elderly patients with EC/EGJC.

\footnotetext{
Abbreviations

AC: Adenocarcinoma; ALP: Alkaline Phosphatase; ALT: Alanine Aminotransferase; AST: Aspartate Aminotransferase; BUN: Blood Urea Nitrogen; cCR: clinical Complete response; CCRT: Concurrent chemoradiotherapy; CGA: Comprehensive Geriatric Assessment; CPR: clinical Partial response; Cr: Creatinine; CRF: Case Report Form; CRR: clinical Response Rate; CT: Computed Tomography; CTCAE: Common Terminology Criteria for Adverse Events; CTD: Collection of Targets-Delineation; CTV: Clinical target volume; dCRT: definitive Concurrent chemoradiotherapy; EC: Esophageal cancer; ECOG: Eastern Cooperative Oncology Group; EGJC: Esophagogastric junction cancer; EQD2: Equivalent Dose in 2 Gy/f; ESMO: European Society for Medical Oncology; GTV-N: Metastatic regional nodes; GTV-T: Gross Tumor Volume; IDMC: Independent Data Monitoring Committee; ITT: Intention to treat; LCR: Local Control Rate; Lt: Lower thoracic; MRI: Magnetic Resonance Imaging; Mt: Middle thoracic; MTD: Maximum tolerated dose; NCCN: National Comprehensive Cancer Network; OAR: Organs at risk; OS: Overall Survival; PGTV: Planning Gross Tumor Volume; PP Per: Protocol; PTV: Planning target volume; RECIST: Response Evaluation Criteria In Solid Tumors; RT: Radiotherapy; RTOG: Radiation Therapy Oncology Group; RTTQA: Radiotherapy Trials Quality Assurance; S-1: Tegafur,Gimeracil and Oteracil Potassium Capsules; SAE: Serious adverse event; SCC: Squamous cell carcinoma; SCLC: Small cell lung cancer; SIB: Simultaneous integrated boost; UNL: Upper Normal Limit; Ut: Upper thoracic
}

\section{Acknowledgements}

We thank all the patients who participated in this trial, all participating branch-centers and investigators who devote their time and passion in the implementation of this study. We thank Jing-Jin-Ji Esophageal and Esophagogastric Cancer Radiotherapy Oncology Group (3JECROG) and Beijing branch of the Chinese Medical Association for the opportunity of initiating this prospective multi-center phase II/III trial. The following list of names show the investigators who contributed this study by making substantial contributions to the delivery of the study: Minghe Li, Miaoling Liu, Kai-Xian Zhang, Ling Li, Yonggang Shi, Nan Bi, Dongfu Chen, Qinfu Feng, Jima Lv, Tao Zhang, Miaomiao Hu, Wei Wang, Ke Liu, Chongli Hao, Haiwen Zhou, Jihong Zhang, Wei Deng, Weiming Han, ect. Tian Yuan gave guiding opinions on the quality control of radiophysics and radiotherapy plans.

\section{Funding}

Beijing Hope Run Special Fund of Cancer Foundation of China (LC2016L04). National Key Projects of Research and Development of China (2016YFC0904600). The funding source has no role in study design, data collection, analysis, interpretation, the writing of the manuscript, or the decision to submit the current study.

\section{Availability of data and materials}

Not applicable - data collection is still ongoing.

\section{Trial status}

The study protocol was approved by the institutional review board in October 2016. Recruitment started in February, 2017 and is currently ongoing.

\section{Authors' contributions}

ZFX and YDZ made substantial contributions to the conception and design of the study, revised the article critically for important intellectual content and gave final approval of the version to be published; XW made contributions to the design of the study, gave substantial contributions to the organization of this trial and revised the article critically; $C L, X M W$ and XW draft the manuscript and equally contributed to the paper; $\mathrm{CH}$, PW, QSP, JQC and XCS participated in designing and conducting the study; LW, YL, XLG, WCZ, WJN, XC made substantial contribution to the delivery of this study and collected data; ZMZ, JL, LD and WQW are currently involved in study implementation. All authors read and approved the final manuscript.

\section{Ethics approval and consent to participate}

Institutional review board approval was obtained for the 3JECROG P-01 trial from the ethical committee of the Chinese Academy of Medical Sciences (reference number NCC2016 YL-06), of Anyang Cancer Hospital (AZLL 022017002170116), of Tianjin Cancer Hospital (E2018309), of Fujian Cancer Hospital (SQ2019-037-01). The 3JECROG P-01 trial is published under NCT02979691 on ClinicalTrials.gov. Written informed consent is obtained from all participants.

\section{Consent for publication}

Not applicable.

\section{Competing interests}

The authors declare that they have no competing interests.

\section{Publisher's Note}

Springer Nature remains neutral with regard to jurisdictional claims in published maps and institutional affiliations.

\footnotetext{
Author details

'Department of Radiation Oncology, National Cancer Center/National Clinical Research Center for Cancer/Cancer Hospital, Chinese Academy of Medical Sciences and Peking Union Medical College, Beijing 100021, China. ${ }^{2}$ Department 4th of Radiation Oncology, Anyang Cancer Hospital, Anyang 455000, China. ${ }^{3}$ Department of Radiation Oncology, the Fourth Hospital of Hebei Medical University, Shijiazhuang 050011, China. ${ }^{4}$ Department of Radiation Oncology, Tianjin Medical University Cancer Institute and Hospital/ National Clinical Research Center for Cancer, Tianjin 300060, China.

${ }^{5}$ Department of Radiation Oncology, Fujian Cancer Hospital/Fujian Medical
} 
University Cancer Hospital, Fuzhou 350014, China. ${ }^{6}$ Department of Radiation Oncology, the First Affiliated Hospital of Nanjing Medical University, Nanjing 210029, China. 'Department of Radiation Oncology, Cancer Hospital Chinese Academy of Medical Sciences, Shenzhen Center, Shenzhen 518000, China.

Received: 25 November 2018 Accepted: 28 March 2019 Published online: 29 April 2019

\section{References}

1. Chen W, Zheng R, Baade PD, Zhang S, Zeng H, Bray F, et al. Cancer statistics in China, 2015. CA Cancer J Clin. 2016;66:115-32.

2. Wu M, van't Veer P, Zhang Z-F, Wang X, Gu X, Han R, et al. A large proportion of esophageal cancer cases and the incidence difference between regions are attributable to lifestyle risk factors in China. Cancer Lett. [internet]. Elsevier Ireland Ltd. 2011;308:189-96 Available from: https://doi.org/10.1016/j.canlet.2011.05.003.

3. Markar SR, Karthikesalingam A, Thrumurthy S, Ho A, Muallem G, Low DE. Systematic review and pooled analysis assessing the association between elderly age and outcome following surgical resection of esophageal malignancy. Dis. Esophagus. 2013;26:250-62 Available from: https://academic.oup.com/dote/ article-lookup/doi/10.1111/j.1442-2050.2012.01353.x.

4. Zehetner J, Lipham JC, Ayazi S, Banki F, Oezcelik A, DeMeester SR, et al. Esophagectomy for cancer in octogenarians. Dis Esophagus. 2010;23:666-9 Available from: https://academic.oup.com/dote/article-lookup/doi/10.1111/j. 1442-2050.2010.01081.x.

5. National Comprehensive Cancer Network: NCCN Clinical Practice Guidelines in Oncology: Esophageal and Esophagogastric Junction Cancers, Version 2, 2018. https://www.nccn.org/professionals/physician_gls/pdf/esophageal.pdf;

6. Lordick F, Mariette C, Haustermans K, Obermannová R, Arnold D. On behalf of the ESMO guidelines committee clinicalguidelines@ESMO org. Oesophageal cancer: ESMO clinical practice guidelines for diagnosis, treatment and followup. Ann. Oncol. 2016;27:v50-7.

7. Li C, Deng W, Wang X, Xiao Z, Zhou Z, Zhang H, et al. A Phase 1/2 Radiation Dose Escalation Trial Using SIB-IMRT Technique With Concurrent Chemotherapy in Unresectable Esophageal Carcinoma. Int. J. Radiat. Oncol. 2017;99:E166 Available from: http://www.sciencedirect.com/science/article/pii//S0360301617 320503?_rdoc=1\&_fmt=high\&_origin=gateway\&_docanchor=\&md5=b84294 49ccfc9c30159a5f9aeaa92ffb.

8. Balducci L, Cohen HJ, Engstrom PF, Ettinger DS, Halter J, Gordon LI, et al. Senior adult oncology clinical practice guidelines in oncology. J Natl Compr Canc Netw Cold Spring Publishing LLC. 2005;3:572-90.

9. Extermann M, Aapro M, Bernabei R, Cohen HJ, Droz J-P, Lichtman S, et al. Use of comprehensive geriatric assessment in older cancer patients: Recommendations from the task force on CGA of the International Society of Geriatric Oncology (SIOG). Crit. Rev. Oncol. Hematol. Elsevier; 2005;55:241-252.

10. Chen CC-H, Kenefick AL, Tang ST, McCorkle R. Utilization of comprehensive geriatric assessment in cancer patients. Crit Rev Oncol Hematol Elsevier. 2004;49:53-67.

11. Wieland D, Hirth V. Comprehensive geriatric assessment. Cancer control, vol. 10. Los Angeles, CA: SAGE PublicationsSage CA; 2003. p. 454-62.

12. Repetto L, Venturino A, Fratino L, Serraino D, Troisi G, Gianni W, et al. Geriatric oncology: a clinical approach to the older patient with cancer. Eur J Cancer Pergamon. 2003;39:870-80.

13. Bernabei R, Venturiero V, Tarsitani P, Gambassi G. The comprehensive geriatric assessment: when, where, how. Crit Rev Oncol Hematol [Internet]. 2000;33:45-56 Available from: http://linkinghub.elsevier.com/retrieve/pii/ S1040842899000487.

14. Balducci L, Extermann M. Management of cancer in the older person: a practical approach. Oncologist AlphaMed Press. 2000;5:224-37.

15. Xiao Z, Zhou Z, Li H. Esophageal Cancer target volume delineation and treatment guidance for radiation therapy (in Chinese). 1st ed. Beijing: People's Medical Publishing House; 2017.

16. Herskovic A, Martz K, Al-Sarraf M, Leichman L, Brindle J, Vaitkevicius V, et al. Combined chemotherapy and radiotherapy compared with radiotherapy alone in patients with Cancer of the esophagus. N Engl J Med. 1992;326: $1593-8$

17. Minsky BD. INT 0123 (radiation therapy oncology group 94-05) phase III trial of combined-modality therapy for esophageal Cancer: high-dose versus standard-dose radiation therapy. J Clin Oncol. 2002;20:1167-74.
18. Tougeron D, Di Fiore F, Thureau S, Berbera N, Iwanicki-Caron I, Hamidou H, et al. Safety and outcome of definitive chemoradiotherapy in elderly. patients with oesophageal cancer. Br. J. Cancer. 2008 [cited 2017 Dec 20];99: 1586-1592. Available from: http://www.ncbi.nlm.nih.gov/pubmed/19002180.

19. Wang H, Li G, Chen L, Duan Y, Zou C, Hu C. Definitive concurrent chemoradiotherapy with S-1 and cisplatin in elderly esophageal squamous cell carcinoma patients. J Thorac Dis. 2017;9:646-54 Available from: http:// jtd.amegroups.com/article/view/12372/10614.

20. Servagi-Vernat S, Créhange G, Roullet B, Guimas V, Maingon P, Puyraveau M, et al. Phase II Study of a Platinum-Based Adapted Chemotherapy Regimen Combined with Radiotherapy in Patients 75 Years and Older with Esophageal Cancer. Drugs Aging. 2015 [cited 2017 Dec 20];32:487-493. Available from: http://link.springer.com/10.1007/s40266-015-0275-8

21. Fukushima M, Sakamoto K, Sakata M, Nakagawa F, Saito H, Sakata Y. Gimeracil, a component of S-1, may enhance the antitumor activity of $X$-ray irradiation in human cancer xenograft models in vivo. Oncol. Rep. 2010 [cited 2017 Dec 19];24:1307-1313. Available from: http://www.ncbi. nlm.nih.gov/pubmed/20878125.

22. Huang J, Cao Y, Wu L, Liao C, He Y, Gao F. S-1-based therapy versus 5-FUbased therapy in advanced gastric cancer: a meta-analysis. Med. Oncol. 2011; 28:1004-11 Available from: http://link.springer.com/10.1007/s12032-010-9594-0.

23. Welsh J, Settle SH, Amini A, Xiao L, Suzuki A, Hayashi Y, et al. Failure patterns in patients with esophageal cancer treated with definitive chemoradiation. Cancer. 2012;118:2632-40 Available from: http://doi.wiley.com/10.1002/ cncr.26586.

24. Song T, Liang X, Fang M, Wu S. High-dose versus conventional-dose irradiation in cisplatin-based definitive concurrent chemoradiotherapy for esophageal cancer: a systematic review and pooled analysis. Expert Rev. Anticancer Ther. Informa UK, Ltd.; 2015;15:1157-1169. Available from: https://doi.org/10.1586/14737140.2015.1074041

25. Welsh J, Palmer MB, Ajani JA, Liao Z, Swisher SG, Hofstetter WL, et al. Esophageal Cancer Dose Escalation Using a Simultaneous Integrated Boost Technique. Int. J. Radiat. Oncol. 2012;82:468-74 Available from: http://linkinghub.elsevier.com/retrieve/pii/S0360301610034449.

26. Zhang W-Z. Simultaneous modulated accelerated radiation therapy for esophageal cancer: A feasibility study. World J. Gastroenterol. 2014;20:13973 Available from: http://www.wjgnet.com/1007-9327/full/v20/i38/13973.htm.

27. Yu W-W, Zhu Z-F, Fu X-L, Zhao K-L, Mao J-F, Wu K-L, et al. Simultaneous integrated boost intensity-modulated radiotherapy in esophageal carcinoma. Strahlentherapie und Onkol. 2014;190:979-86 Available from: http://link. springer.com/10.1007/s00066-014-0636-y.

28. Yu W, Cai X-W, Liu Q, Zhu Z-F, Feng W, Zhang Q, et al. Safety of dose escalation by simultaneous integrated boosting radiation dose within the primary tumor guided by 18FDG-PET/CT for esophageal cancer. Radiother. Oncol. Elsevier Ireland Ltd; 2015;114:195-200. Available from: https://doi.org/ 10.1016/.j.radonc.2014.12.007

29. Chen J, Guo H, Zhai T, Chang D, Chen Z, Huang R, et al. Radiation dose escalation by simultaneous modulated accelerated radiotherapy combined with chemotherapy for esophageal cancer: a phase II study. Oncotarget. 2016;7:22711-9 Available from: http://www.oncotarget.com/abstract/8050.

30. Welsh JW, Seyedin SN, Allen PK, Hofstetter WL, Ajani JA, Chang JY, et al. Local control and toxicity of a simultaneous integrated boost for dose escalation in locally advanced esophageal Cancer: interim results from a prospective phase 1/I trial. J. Thorac. Oncol. Elsevier Ltd; 2017;12:375-382. Available from: https://doi.org/10.1016/j.jtho.2016.10.013

31. Cohen HJ, Feussner JR, Weinberger M, Carnes M, Hamdy RC, Hsieh F, et al. A controlled trial of inpatient and outpatient geriatric evaluation and management. N. Engl. J. Med. 2002;346:905-12 Available from: http://www. nejm.org/doi/abs/10.1056/NEJMsa010285.

32. Corre R, Greillier L, Le Caër H, Audigier-Valette C, Baize N, Bérard H, et al. Use of a Comprehensive Geriatric Assessment for the Management of Elderly Patients With Advanced Non-Small-Cell Lung Cancer: The Phase III Randomized ESOGIA-GFPC-GECP 08-02 Study. J. Clin. Oncol. 2016;34:1476-83 Available from: http://ascopubs.org/doi/10.1200/JCO.2015.63.5839.

33. Caillet P, Canoui-Poitrine F, Vouriot J, Berle M, Reinald N, Krypciak S, et al. Comprehensive geriatric assessment in the decision-making process in elderly patients with cancer: ELCAPA study. J Clin Oncol. 2011;29:3636-42 Available from: http://ascopubs.org/doi/10.1200/JCO.2010.31.0664.

34. Aaronson NK, Ahmedzai S, Bergman B, Bullinger M, Cull A, Duez NJ, et al. The European Organization for Research and Treatment for use in international clinical trials in oncology. J Natl Cancer Inst. 1993;85:365-76. 
35. Blazeby JM, Conroy T, Hammerlid E, Fayers P, Sezer O, Koller M, et al. Clinical and psychometric validation of an EORTC questionnaire module, the EORTC QLQ-OES18, to assess quality of life in patients with oesophageal cancer. Eur J Cancer. 2003:39:1384-94.

36. Oken MM, Creech RH, Tormey DC, Horton J, Davis TE, McFadden ET, et al. Toxicity and response criteria of the eastern cooperative oncology group. Am. J. Clin. Oncol. 1982;5:649-55 Available from: http://www.ncbi.nlm.nih. gov/pubmed/7165009.

37. Colin Munro MacLeod. The clinical evaluation of chemotherapeutic agents in cancer, in Macleod CM(ed): Evaluation of Chemotherapeutic Agents. New York: Columbia University Press; 1948.

38. Mahoney Fl, Barthel DW. Functional Evaluation: the Barthel Index. Md. State Med. J. 1965;14:56-61 Available from: http://www.strokecenter.org/wpcontent/uploads/2011/08/barthel_reprint.pdf.

39. Lawton MP, Brody EM. Assessment of older people: self-maintaining and instrumental activities of daily living. Gerontologist. 1969;9:179-86 Available from: http://gerontologist.oxfordjournals.org/cgi/doi/10.1093/geront/9.3 Part_1.179.

40. Charlson ME, Pompei P, Ales KL, MacKenzie CR. A new method of classifying prognostic comorbidity in longitudinal studies: development and validation. J Chronic Dis. 1987;40:373-83.

41. Folstein MF, Folstein SE, McHugh PR. "Mini-mental state". A practical method for grading the cognitive state of patients for the clinician. J Psychiatr Res. 1975;12:189-98.

42. Hoyl MT, Alessi CA, Harker JO, Josephson KR, Pietruszka FM, Koelfgen M, et al. Development and testing of a five-item version of the geriatric depression scale. J. Am. Geriatr. Soc. 1999;47:873-8 Available from: http:// www.ncbi.nlm.nih.gov/pubmed/10404935.

43. Landi F. Body Mass Index and Mortality Among Hospitalized Patients. Arch. Intern. Med. 2000;160:2641 Available from: http://archinte.jamanetwork.com/ article.aspx?doi=10.1001/archinte.160.17.2641.

44. Guigoz $Y$, Vellas B. The mini nutritional assessment (MNA) for grading the nutritional state of elderly patients: presentation of the MNA, history and validation. Nestle Nutr. Workshop Ser. Clin. Perform. Programme. 1999;1:3-12.

45. Sherbourne CD, Stewart AL. The MOS social support survey. Soc Sci Med. 1991;32:705-14.

Ready to submit your research? Choose BMC and benefit from:

- fast, convenient online submission

- thorough peer review by experienced researchers in your field

- rapid publication on acceptance

- support for research data, including large and complex data types

- gold Open Access which fosters wider collaboration and increased citations

- maximum visibility for your research: over $100 \mathrm{M}$ website views per year

At $\mathrm{BMC}$, research is always in progress.

Learn more biomedcentral.com/submissions 